\title{
Measurements of heavy flavor properties at CMS
}

\author{
Po-Hsun Chen ${ }^{* \dagger}$ \\ National Taiwan University \\ E-mail: pohsun.chen.hepegmail.com
}

A new decay channel $B_{S 2}(5840)^{0} \rightarrow B^{0} K_{S}$ has been observed by $6.3 \sigma$ to $7.0 \sigma$. Accompanied with the obserservation, there is also a hint of $B_{s 1} \pm B^{0} K_{s}$. Corresponding branching fraction ratio to $B^{()+} K^{-}$channel is calculated to be $R_{2}^{0 \pm}=0.432 \pm 0.077$ (stat $) \pm 0.075$ (syst. $) \pm 0.021(P D G)$ and $R_{1}^{0 \pm}=0.49 \pm 0.12$ (stat) \pm 0.07 (syst. $) \pm 0.02(P D G)$. In the study of $\Lambda_{b} \rightarrow J / \psi \Lambda$ decay, all there decay parameters of interest show consistency with LHCb and ATLAS results. However, the measured parity asymmetry parameter1disagree with the HQET prediction by over 5 standard deviation. Standard lifetime measurement for $B^{0}, B_{s}, B_{c}^{+}, \Lambda_{b}$ matches fine with the world-averaged values. The CMS result for $B_{c}^{+}$is in favor of the $\mathrm{LHCb}$ result to those derived at Tevatron.

ICHEP 2018, XXXIX International Conference on High Energy Physics

4-11 July 2018

Seoul, Korea

* Speaker.

${ }^{\dagger}$ On behalf of the CMS Collaboration 


\section{Measurements}

This letter provides a review of B hadron properties research conducted with the CMS experiment at the Large Hadron Collidor (LHC). All data is collected in proton-proton collision at the center-of-mass energy of 7TeV $\left(5.2 \mathrm{fb}^{-1}\right)$ and that of $8 \mathrm{TeV}\left(19.6 \mathrm{fb}^{-1}\right)$.

\subsection{Studies of $\mathbf{P}$ wave excited states of $B_{S}$ and the observation of $B_{s 2}(5840)^{0} \rightarrow B^{0} K_{S}$ decay}

In a $B_{s}^{0}$ bound state, since the bottom quark is heavier than the strange quark, the system is suitable to be described with the heavy quark effective theory (HQET).Several orbitally excited states of $B_{s}^{0}$ meson has seen through $B^{(*)+} K^{-}$decay channel. According to HQET, there is no significant difference to replace $B^{(*)+} K^{-}$with $B^{(*) 0} K_{s}$. It indeed replaces the $u \bar{u}$ pair with a $d \bar{d}$ pair in the decay. In figure $\mathbb{U}$, first observation of the $B_{s 2}^{*} \rightarrow B^{0} K_{s}$ and the evidence of $B_{s 1}^{*} \rightarrow B^{*} K_{s}$, in which $B^{*} \rightarrow B^{0} \gamma, B^{0} \rightarrow J / \psi(\rightarrow \mu \mu) K^{* 0}\left(\rightarrow K^{+} \pi^{-}\right)$, are presented using the 8TeV data. [1] The significance of the $B_{s 2}^{*} \rightarrow B^{0} K_{s}$ decay is calculated to be in the range of 6.3 to 7.0 standard deviation, and it's 3.6 to 3.9 standard deviation for $B_{s 1} \rightarrow B^{* 0} K_{s}$. The corresponding branching fraction ratios of $B^{0} K_{s}$ channel to $B^{(*)+}\left(\rightarrow J / \psi K^{+}\right) K^{-}$channel are provided:

$$
\begin{aligned}
& R_{2}^{0 \pm}=\frac{\mathscr{B}\left(B_{s 2}^{*} \rightarrow B^{0} K_{s}\right)}{\mathscr{B}\left(B_{s 2}^{*} \rightarrow B^{+} K^{-}\right)}=0.432 \pm 0.077 \text { (stat) } \pm 0.075 \text { (syst.) } \pm 0.021(\mathrm{PDG}) \\
& \left.R_{1}^{0 \pm}=\frac{\mathscr{B}\left(B_{s 1} \rightarrow B^{* 0} K_{s}\right)}{\mathscr{B}\left(B_{s 1} \rightarrow B^{*+} K^{-}\right)}=0.49 \pm 0.12 \text { (stat) } \pm 0.07 \text { (syst. }\right) \pm 0.02(\mathrm{PDG})
\end{aligned}
$$

in which the branching fraction $\mathscr{B}\left(B^{*(+)} \rightarrow B^{(+)} \gamma\right)$ is assumed to be $100 \%$.
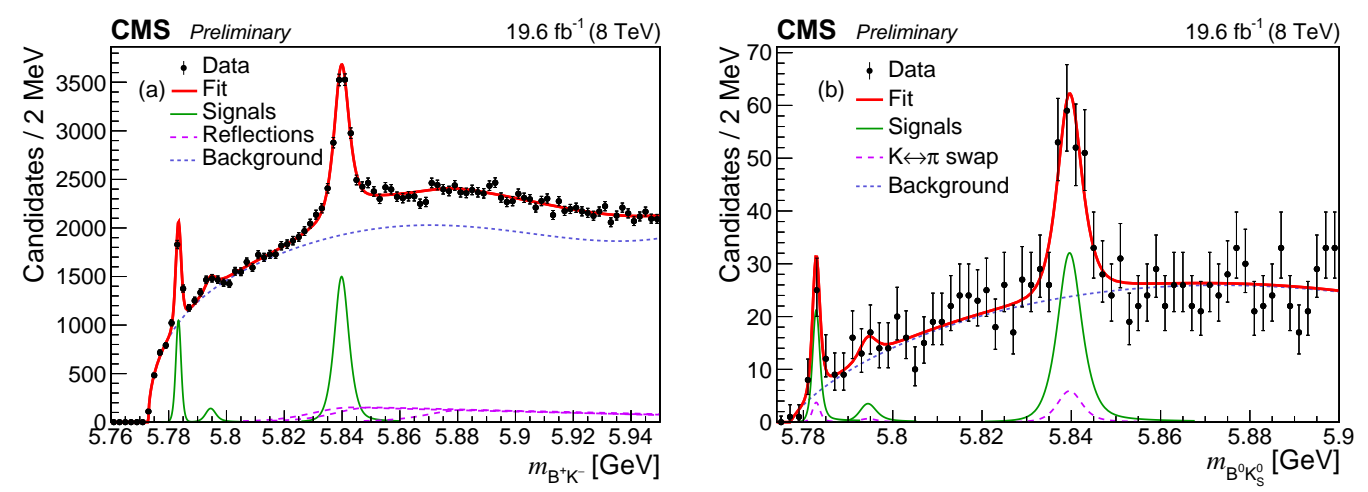

Figure 1: The fitting result to invariant mass spectrum of $B^{+} K^{-}$and $B^{0} K_{s}$, where $m_{B K}=M(B K)-M(B)+$ $M_{B}^{P D G}$ is used to improve mass resolution. In $B^{+} K^{-}$spectrum, the reflection term comes from the excited $B^{0}$ meson decays. While in $B^{0} K_{S}$ spectrum, the long-dashed line denotes the swapping of $K^{ \pm} \rightarrow \pi^{ \pm}$in the $B^{0}$ reconstruction.

\section{$1.2 \Lambda_{b}$ polarization and angular parameters in $\Lambda_{b} \rightarrow J / \psi \Lambda$}

The $\Lambda_{b}$ decays to $J / \psi \Lambda$ via a $b \rightarrow s$ loop, which for long time is treated as a good source of QCD study. For this decay process, assuming uniform detector acceptance over the azimuthal angles, the angular distribution of decay products can be expressed with five parameters [2] and we 
Table 1: The measured polarization, parity asymmetry with the corresponding statistical error (former) and systematic error (latter) in comparison with theoretical [4-6] and other experimental results [7,8]. Remark that notation of $\alpha_{b}$ used in ATLAS, LHCb, and theoretical papers is equal to $-\alpha_{1}$. As for $\alpha_{2}$, given the reference papers don't provide the value directly, the values are calculated from helicity amplitude provided in common and found to be equivalent to -1 .

\begin{tabular}{ccccc}
\hline Parameter & CMS & LHCb & ATLAS & Prediction \\
\hline \hline$P$ & $0.00 \pm 0.06 \pm 0.06$ & $0.06 \pm 0.07 \pm 0.02$ & - & $0.1 \sim 0.2$ \\
\hline$-\alpha_{1}$ & $-0.14 \pm 0.14 \pm 0.10$ & $0.05 \pm 0.17 \pm 0.07$ & $0.30 \pm 0.16 \pm 0.06$ & $-0.2 \sim-0.1$ \\
& & & & 0.78 (HQET) \\
\hline$\alpha_{2}$ & $-1.11 \pm 0.08 \pm 0.11$ & -1 & -1 & -
\end{tabular}

have most interest in 3 meaningful ones: $P$, the $\Lambda_{b}$ transverse polarization, $\alpha_{1}$, the parity asymmetry parameter for the $\Lambda_{b} \rightarrow J / \psi \Lambda$ decay, and $\alpha_{2}$, the $\Lambda$ longitudinal polarization. We performed the angular analysis [3] using both $7 \mathrm{TeV}$ and $8 \mathrm{TeV}$ data. The results is summarized in Table $\mathrm{W}$. All experimental resuls is in consistency, while the HQET predicted $\alpha_{1}$ is disfavored by over $5 \sigma$.

\subsection{Lifetime of $\mathbf{b}$ hadrons in decays with $J / \psi$}

The $8 \mathrm{TeV}$ collision data triggered by events with a $J / \psi$ candidate is used, and the result is summarized in Table $\square$. For $B^{0}$ and $\Lambda_{b}$ decays, we perform unbinned maximum likelihood fit to the mass spectrum, lifetime $(c \tau)$ to extract all the needed information. In the case of $B_{s}$, due to the non-negligible contribution from $B^{+} \rightarrow J / \psi K^{+}$, extended fit is adopted. We indirectly measure the $B_{c}^{+}$lifetime on the basis of the precisely known $B^{+}$lifetime from the difference of total decay width.

Table 2: Summary of measured $c \tau$ in comparison with PDG values [9]. Note that the former uncertainty term stands for statistical uncertainty, while the latter stands for systematic uncertainty.

\begin{tabular}{lll}
\hline Channel & CMS result & PDG value \\
\hline \hline$B^{0} \rightarrow J / \psi K^{* 0}$ & $453.0 \pm 1.6 \pm 1.8 \mu \mathrm{m}$ & $456.0 \pm 1.2 \mu \mathrm{m}$ \\
$B^{0} \rightarrow J / \psi K_{s}$ & $457.8 \pm 2.7 \pm 2.8 \mu \mathrm{m}$ & $456.0 \pm 1.2 \mu \mathrm{m}$ \\
$\Lambda_{b} \rightarrow J / \psi \Lambda$ & $442.9 \pm 8.2 \pm 2.8 \mu \mathrm{m}$ & $441.0 \pm 3.0 \mu \mathrm{m}$ \\
$B_{s}^{0} \rightarrow J / \psi \pi^{+} \pi^{-}$ & $502.7 \pm 10.2 \pm 3.4 \mu \mathrm{m}$ & $497.4 \pm 9.6 \mu \mathrm{m}$ \\
$B_{s}^{0} \rightarrow J / \psi \phi(1020)$ & $443.9 \pm 2.0 \pm 1.5 \mu \mathrm{m}$ & $443.7 \pm 3.6 \mu \mathrm{m}$ \\
$B_{c}^{+} \rightarrow J / \psi \pi^{+}$ & $162.3 \pm 7.8 \pm 4.2 \mu \mathrm{m}$ & $152.7 \pm 2.7 \mu \mathrm{m}$ \\
\hline
\end{tabular}

As a result, the CMS measurements are all in good match with the world-averaged values. Our measured $B_{c}^{+}$lifetime, presented in $c \tau$, is in favor of the LHCb result $(153.9 \pm 3.3 \mu \mathrm{m})$ [10] to the Tevatron results $(\sim 135 \mu \mathrm{m})[11,12]$.

\section{Conclusion}

A new decay channel $B_{s 2}^{*}(5840)^{0} \rightarrow B^{0} K_{s}$ has been observed by 6.3 to 7.0 standard deviation. Accompanied with the observation, there is also a hint of $B_{s 1} \rightarrow B^{* 0} K_{s}$. Corresponding branching fraction ratio to $B^{(*)+} K^{-}$channel is calculated to be $R_{2}^{0 \pm}=0.432$ and $R_{1}^{0 \pm}=0.49$. In the study of 
$\Lambda_{b} \rightarrow J / \psi \Lambda$ decay, all the decay parameters of interest show consistency with LHCb and ATLAS results. However, the measured parity asymmetry parameter $\alpha_{1}$ disagree with the HQET prediction by over 5 standard deviation. Standard lifetime measurement for $B^{0}, B_{s}, B_{c}^{+}, \Lambda_{b}$ matches fine with the world-averaged values. The CMS result for $B_{c}^{+}$is in favor of the $\mathrm{LHCb}$ result to those derived at Tevatron.

Property study of B hadrons has played a must-have role in understanding QCD. The CMS B physics analysis group has brought numerous results from LHC Run-1 data and foreseen updates with run-II data will lead to deeper insights to the physics picture.

\section{References}

[1] CMS Collaboration, "Studies of $\mathrm{B}_{\mathrm{s} 2}^{*}(5840)^{0}$ and $\mathrm{B}_{\mathrm{s} 1}(5830)^{0}$ mesons including the observation of the $\mathrm{B}_{\mathrm{s} 2}^{*}(5840)^{0} \rightarrow \mathrm{B}^{0} \mathrm{~K}_{\mathrm{S}}^{0}$ decay in proton-proton collisions at $\sqrt{s}=8 \mathrm{TeV}$ ", Submitted to: Eur. Phys. J. (2018) arxiv: 1809.03578 .

[2] M. Kramer and H. Simma, “Angular correlations in Lambda/b $\rightarrow$ Lambda + V: Polarization measurements, HQET and CP violation”, Nucl. Phys. Proc. Suppl. 50 (1996) 125-129, doi:10.1016/0920-5632(96)00378-7. [,125(1996)].

[3] CMS Collaboration, "Measurement of the $\Lambda_{b}$ polarization and angular parameters in $\Lambda_{b} \rightarrow J / \psi \Lambda$ decays from pp collisions at $\sqrt{s}=7$ and 8 TeV”, Phys. Rev. D97 (2018), no. 7, 072010, doi:10.1103/PhysRevD.97.072010, arxiv:1802.04867.

[4] G. Hiller, M. Knecht, F. Legger, and T. Schietinger, "Photon polarization from helicity suppression in radiative decays of polarized Lambda(b) to spin-3/2 baryons", Phys. Lett. B649 (2007) 152-158, doi:10.1016/j.physletb.2007.03.056, arXiv:hep-ph/0702191.

[5] Z. J. Ajaltouni, E. Conte, and O. Leitner, "Lambda(b) decays into Lambda-vector", Phys. Lett. B614 (2005) 165-173, doi:10.1016/j.physletb.2005.04.014, arXiv:hep-ph/0412116.

[6] O. Leitner, Z. J. Ajaltouni, and E. Conte, “An Angular distribution analysis of Lambda(b) decays", Nucl. Phys. A755 (2005) 435-438, doi:10.1016/j.nuclphysa.2005.03.051, arXiv:hep-ph/0412131.

[7] ATLAS Collaboration, "Measurement of the parity-violating asymmetry parameter $\alpha_{b}$ and the helicity amplitudes for the decay $\Lambda_{b}^{0} \rightarrow J / \psi+\Lambda^{0}$ with the ATLAS detector", Phys. Rev. D89 (2014), no. 9, 092009, doi:10.1103/PhysRevD.89.092009, arxiv:1404.1071.

[8] LHCb Collaboration, "Measurements of the $\Lambda_{b}^{0} \rightarrow J / \psi \Lambda$ decay amplitudes and the $\Lambda_{b}^{0}$ polarisation in $p p$ collisions at $\sqrt{s}=7 \mathrm{TeV}$ ", Phys. Lett. $\mathbf{B 7 2 4}$ (2013) 27-35, doi:10.1016/j.physletb.2013.05.041, arXiv:1302.5578.

[9] Particle Data Group Collaboration, "Review of Particle Physics", Chin. Phys. C40 (2016), no. 10, 100001, doi: 10.1088/1674-1137/40/10/100001.

[10] LHCb Collaboration, "Measurement of the lifetime of the $B_{c}^{+}$meson using the $B_{c}^{+} \rightarrow J / \psi \pi^{+}$decay mode", Phys. Lett. B742 (2015) 29-37, doi:10.1016/7.physletb.2015.01.010, arxiv: 1411.6899 .

[11] D0 Collaboration, "Measurement of the lifetime of the $B_{c}^{ \pm}$meson in the semileptonic decay channel", Phys. Rev. Lett. 102 (2009) 092001, doi:10.1103/PhysRevLett.102.092001, arxiv:0805.2614.

[12] CDF Collaboration, "Measurement of the $B_{c}^{-}$meson lifetime in the decay $B_{c}^{-} \rightarrow J / \psi \pi^{-}$", Phys. Rev. D87 (2013), no. 1, 011101, doi:10.1103/PhysRevD.87.011101, arXiv: 1210.2366. 